\title{
LHomme
}

LHOMME Revere trancease deanthropologie

$189 \mid 2009$

Oralité et écriture

\section{Helle Johannessen \& Imre Lazar, eds, Multiple Medical Realities \\ Nicole Durisch Gauthier, Ilario Rossi \& Jörg Stolz, eds, Quêtes de santé}

Samuel Lézé

\section{OpenEdition}

\section{Journals}

Édition électronique

URL : http://journals.openedition.org/lhomme/28806

DOI : 10.4000/lhomme.28806

ISSN : 1953-8103

Éditeur

Éditions de l'EHESS

Édition imprimée

Date de publication : 1 janvier 2009

Pagination : 307-309

ISSN : 0439-4216

Référence électronique

Samuel Lézé, « Helle Johannessen \& Imre Lazar, eds, Multiple Medical Realities

Nicole Durisch Gauthier, Ilario Rossi \& Jörg Stolz, eds, Quêtes de santé », L'Homme [En ligne], 189|

2009, mis en ligne le 03 janvier 2017, consulté le 24 septembre 2020. URL : http://

journals.openedition.org//homme/28806 ; DOI : https://doi.org/10.4000/lhomme.28806

Ce document a été généré automatiquement le 24 septembre 2020.

(c) École des hautes études en sciences sociales 


\title{
Helle Johannessen \& Imre Lazar, eds, Multiple Medical Realities Nicole Durisch Gauthier, Ilario Rossi \& Jörg Stolz, eds, Quêtes de santé
}

\author{
Samuel Lézé
}

\section{RÉFÉRENCE}

Helle JOHANNESSEN \& Imre LAZAR, eds, Multiple Medical Realities. Patients and Healers in Biomedical, Alternative and Traditional Medecine, New York-Oxford, Berghahn Books, 2006, 202 p., notes bibliogr., index, fig. («The EASA Series » 4).

Nicole DuRISCh GAUTHIER, Ilario Rossi \& Jörg StoLZ, eds, Quêtes de santé. Entre soins médicaux et guérisons spirituelles, Genève, Labor \& Fides, 2007, 140 p., bibl.

CES DEUX OUVRAGES sont consacrés à l'étude du pluralisme médical. Depuis les travaux pionniers de Charles Leslie ${ }^{1}$, la majorité des recherches sur ce thème a été menée au sein de systèmes médicaux asiatiques ou africains, où coexistent médecine cosmopolitaine et savoirs thérapeutiques locaux. Mais paradoxalement, à l'heure du triomphe de la biomédecine, ce phénomène est désormais généralisé : le champ de la médecine officielle (en Europe et Amérique du Nord) est concurrencé par de nombreuses pratiques "alternatives» et "traditionnelles». C'est pourquoi, dans ce contexte de globalisation et de prolifération des modèles de santé, une large place est donnée à des études de cas sur l'homéopathie, le néochamanisme et d'autres pratiques religieuses sur des terrains géographiquement très divers (Hongrie, Ghana, Allemagne, GrandeBretagne, Chine, Asie du Sud, États-Unis, Canada, Équateur et exclusivement la Suisse pour le second ouvrage collectif). 
2 Le volume 4 de l'EASA ${ }^{2}$ montre les deux principaux enjeux de cette investigation. Le premier, empirique, consiste à analyser les relations, tensions et confrontations des approches thérapeutiques, à décrire la complexité du recours et la façon dont les acteurs associent de «multiples réalités médicales». En agissant dans plusieurs mondes, comment se combinent en effet des conceptions différenciées du corps, de la santé et de la guérison? Dans sa préface, Thomas Csordas dégage quatre relations idéales typiques pour penser les conditions de possibilités du pluralisme : compétition ou concurrence, complémentarité (une solution différente pour un trouble), coordination (une solution pour un trouble différent) et coexistence pacifique. Autant de pistes qui se trouvent au principe des contributions empiriques de chacun des auteurs dans les deux grandes parties (l'organisation sociale de l'offre de soin et l'expérience de guérison des patients) de ce volume.

3 À cette complexité des idiomes disponibles comme des répertoires stratégiques répondent une représentation de soi plus flexible et une perception du monde renouvelée. D'où le second enjeu, plus théorique, qui est une tentative de formuler un programme heuristique pour dépasser l'alternative entre approche phénoménologique et approche structurelle, en mobilisant dans l'analyse toutes les échelles du corps (social, vécu, politique). Le risque de l'entreprise, auquel les auteurs s'exposent, est de proposer une sorte d'approche bio-psychosociale holistique (plutôt que véritablement holiste au sens anthropologique du terme), équivalent du modèle valorisé par bien des médecines officielles (oMs) comme alternative (homéopathie), qui aboutirait finalement à un pluralisme théorique et éclectique qui ne convainc pas réellement de sa cohérence théorique ni même de sa pertinence. Entre Scheper-Hughes, Lock, Bourdieu, LéviStrauss et Bruno Latour, on saisit mal la compatibilité de ces approches. Du coup, un doute ne manque pas d'assaillir le lecteur. Pourquoi tant d'énergie déployée pour concilier ces dimensions plutôt que de reformuler intégralement un cadre d'analyse plus respectueux des multiples dimensions de la réalité sociale initialement séparée par l'analyse?

Le second volume, Quêtes de santé, fruit d'un colloque organisé par le Centre intercantonal d'information sur les croyances (CIC) à l'Université de Genève, en 2006, s'inscrit dans une série d'études francophones sur le pluralisme médical ${ }^{3}$. Il interroge les effets de concurrence ou de complémentarité possibles des offres thérapeutiques dans le contexte très circonscrit de la Suisse, mais offre un regard pluridisciplinaire qui a la caractéristique de réunir, outre des chercheurs en sciences sociales (anthropologie, histoire, sociologie), des acteurs de la santé (médecins, juristes). Ces derniers s'interrogent sur les critères de démarcation épistémologique (Bertrand Kiefer) ou juridique (François Bellanger, Béatrice Despland) entre médecines officielles et offres de guérison. La présidente du cIC (Nicole Durish-Gauthier) expose un essai de typologie des mouvements religieux et l'information que délivre le centre au grand public. La difficulté est ici l'identification administrative et économique de ces offres: clarifier l'opacité d'un marché des biens de salut personnel en pleine croissance. En la matière, l'État suisse recense, mais ne contrôle pas. Le problème est aussi l'auto-identification et la quête de légitimité de ces mouvements religieux en termes de science ou de psychothérapie plutôt qu'en termes de «religion». Pour Thomas Sandoz, ce jeu d'identification et de contre-identification (opposition et concurrence) est la raison de l'identité, du succès et de la pérennité historique de ces pratiques. Étrange truisme que d'expliquer le succès d'une offre alternative par le fait même d'être alternative! 
Étrange histoire qui ne cesse de répéter le même jeu! Comment comprendre alors la spécificité actuelle du phénomène?

Des éléments de réponse sont à glaner dans les articles des chercheurs en sciences sociales même si, dans sa conclusion, Jean Benoist réitère une démarcation qui tente de dépasser l'opposition ou la concurrence des offres sous une forme de complémentarité entre technique (médicale) et symbolique. Comme si les mouvements religieux et postpsychanalytiques se cantonnaient à offrir des biens de saluts et non des techniques de soins... Pour Ilario Rossi, la convergence des sphères (médicale et religieuse), dont la fracture est pourtant fondatrice de la modernité, est un analyseur de la reconfiguration de la place du patient dans le système de santé, de la réhumanisation de la médecine et des nouveaux engagements religieux marqués par l'omniprésence et la force sociale de la valeur Santé. Ce dernier point est illustré par Véronique Altglas dans son étude d'un cas de psychologisation de la religion. Elle montre, d'une part, l'alignement du mouvement néo-oriental Siddha Yoga sur celui, psychothérapeutique, de la contreculture américaine et, en restituant le parcours de deux disciples, décrit leurs soucis de pragmatisme, d'efficacité et de développement

6 de soi. En étudiant la guérison en action dans le cadre d'un atelier pentecôtiste en Suisse, l'article de Jorg Stolz a également le mérite d'analyser les conditions pragmatiques d'un régime dévotionnel charismatique : comment rend-il plausibles la perception d'un miracle et la production d'un nouvel état? Il décrit ainsi les règles performatives d'un jeu de langage spécifique manié par le guérisseur au cours d'une séance collective.

7 En somme, on a tout de même le sentiment que ces ouvrages manquent l'essentiel :1) expliquer le phénomène social du pluralisme en tant que tel plutôt que d'en décrire des aspects, certes très suggestifs, mais partiels ; 2) analyser les évolutions sociales de l'autorité de la médecine et sa perte d'autonomie, plutôt que ses effets et la prolifération fascinante d'offres de guérison en tout genre. Il s'agit alors, comme l'a suggéré de longue date Eliot Freidson de déplacer son regard sur la structure du système de santé et ses bouleversements depuis les années 1970. Si l'on suit cette analyse anthropologique au sens fort du terme, il convient d'interroger la façon dont la " nouvelle gouvernance " gestionnaire du système de santé a ouvert la porte à la quête personnelle de guérison et à l'offre de charisme thérapeutique, en subordonnant l'autorité cognitive de la médecine à l'autorité légale de la bureaucratie et la clinique du cas à l'ordre désingularisant de la preuve statistique.

\section{NOTES}

1. À signaler l'hommage de Mark Nichter \& Margaret Lock, eds, New Horizons in Medical Anthropology. Essays in Honour of Charles Leslie, New York-London, Routledge, 2002

2. European Association of Social Anthropologists. 


\section{AUTEUR}

\section{SAMUEL LÉZÉ}

Institut de recherche interdisciplinaire sur les enjeux sociaux, Paris.

Samuel.Leze@ens.fr 